\title{
Expression of miR-136 is associated with the primary cisplatin resistance of human epithelial ovarian cancer
}

\author{
HENAN ZHAO $^{1 *}$, SHA LIU $^{1,2^{*}}$, GUANG WANG $^{1}$, XIAN WU $^{1}$, YANFANG DING $^{1}$, \\ GORDON GUO $^{3}$, JIYONG JIANG ${ }^{2}$ and SHIYING CUI ${ }^{1}$
}

\author{
${ }^{1}$ Dalian Medical University, Dalian, Liaoning 116044; ${ }^{2}$ Obstetrics and Gynecology Hospital, Dalian, Liaoning 116003 , \\ P.R. China; ${ }^{3}$ Department of Radiation Oncology, University of Manitoba, Winnipeg, MB R37 2N2, Canada
}

Received July 2, 2014; Accepted August 12, 2014

DOI: $10.3892 /$ or.2014.3640

\begin{abstract}
MicroRNAs (miRNAs) are involved in regulating the response of cancer cells to various therapeutic interventions, yet their involvement in the chemoresistance of human epithelial ovarian cancer is not fully understood. We found that miR-136 was significantly downregulated in specimens from patients with chemoresistant epithelial ovarian cancer. In the present study, we aimed to clarify the role of miR-136 in regulating the chemoresistance of ovarian cancer. Thirtyfour tumor bank specimens and 2 well-established human ovarian cancer cell lines, C13 and OV2008, were used. We found that miR-136 expression was significantly reduced in primary platinum-resistant patients and the ovarian cancer OVC cell line. Enforced expression of miR-136 decreased the chemoresistance to cisplatin in OVC cells through inhibition of cell survival. In addition, we found no association between miR-136 and migration or invasion potential in the ovarian cancer cell lines. However, in the platinum-resistant C13 cell line, the overexpression of miR-136 markedly promoted an apoptotic response to cisplatin. Furthermore, the levels of adducts corrected with their extent of DNA damage/repair, in terms of the percentage of DNA in comet tails, tail length, tail moment (TM), and olive tail moment (OTM), revealed that miR-136 is essential for the repair of cisplatin-induced DNA damage. Our findings suggest that miR-136 may function as an anti-oncogene and deficiency of miR-136 expression in ovarian cancer can induce chemoresistance at least in part
\end{abstract}

Correspondence to: Professor Shiying Cui, Dalian Medical University, 9 LvShun South Road-W, Dalian, Liaoning 116044, P.R. China

E-mail: scui2003@dlmedu.edu.cn

Professor Jiyong Jiang, Obstetrics and Gynecology Hospital, Dalian, Liaoning 116003, P.R. China

E-mail: jjyd159@163.com

*Contributed equally

Key words: ovarian cancer, serous carcinoma, microRNA, chemoresistance, personalized treatment by downregulating apoptosis and promoting the repair of cisplatin-induced DNA damage. Thus, miR-136 may provide a biomarker for predicting the chemosensitivity to cisplatin in patients with epithelial ovarian cancer.

\section{Introduction}

Epithelial ovarian cancer (EOC) is the most lethal gynecologic malignancy (1-3) which caused an estimated 15,520 deaths in the USA in 2008 (2). The standard treatment protocol used in the initial management of advanced-stage ovarian cancer is cytoreductive surgery, followed by primary chemotherapy with a platinum-based regimen that usually includes cisplatin. Although the management of ovarian cancer has improved over the last 20 years due to more effective surgical techniques and optimized combinational chemotherapy, the overall cure rate is only $30 \%$. One major limitation to the successful treatment of ovarian cancer is the resistance to the currently preferred chemotherapeutic regimen of platinum plus paclitaxel. Empiric-based treatment strategies are used and result in many patients with chemotherapy-resistant disease receiving multiple cycles of often toxic therapy without success before the lack of efficacy is identified. Therefore, the ability to predict the response to and the mechanism involved in platinumbased primary therapies is a major step towards improving the outcome and individualizing treatments for women with ovarian cancer.

In the past few years, research has focused on the discovery of a new class of genomic regulators which are now universally recognized as central players in virtually the development and progression of all neoplasms, named microRNAs (miRNAs). miRNAs are short noncoding RNAs that act as post-transcriptional regulators. They have wide-ranging roles in diverse biological processes, such as development, differentiation, growth and apoptosis (4). miRNAs may also exert a role either as an oncogene or a tumor suppressor by affecting the response to various therapeutic interventions $(5,6)$. In recent years, studies have highlighted the relationship between miRNAs and the resistance to chemotherapy $(5,7,8)$.

Based on miRNA microarray data in our previous research, miR-136 was found to be significantly downregulated in chemoresistant ovarian cancer specimens. To understand the mechanism of miR-136 in regulating chemoresistance, we 
applied this approach to identify miRNA expression patterns within primary EOC that may predict the response to primary platinum-based chemotherapy. Coupled with this, we analyzed the bio-functional variations that reflect and identify the regulation of various oncogenic-miRNA signaling pathways to identify unique mechanisms of platinum-resistance that can guide the use of chemotherapy drugs in platinum-resistant EOC patients.

\section{Materials and methods}

Patient samples and cell lines. We evaluated a total of 34 EOC tissue samples that were resected at the time of primary surgery from patients who went on to receive platinum-based chemotherapy. The clinicopathological characteristics of the patients who contributed the OVC samples are listed in Table I. All tissue samples were collected at the Obstetrics and Gynecology Hospital, Dalian, China between July 2004 and November 2010. The study was approved by the Institutional Review Board of the Ministry of Science and Technology of China [China Human Resource Management Office (2009) 015]. Two independent pathologists with no knowledge of the patient clinical data reviewed the pathological specimens before they were formalin-fixed and paraffin-embedded (FFPE). The cases were classified according to the FIGO staging system. A complete response (CR) was classified as the complete disappearance of all measurable and assessable disease or, in the absence of measurable lesions, a normalization of CA-125 levels following platinum-based therapy. An incomplete response (IR) was defined as only a partial response, no response, or progression during primary therapy (9). CA-125 response criteria were based on established guidelines and were used only in the cases with the absence of a measurable lesion (9-11).

Experiments were performed with a group of human EOC cell lines, including one cisplatin-sensitive parental cell line (OV2008) and its cisplatin-resistant variant (C13) $(1,12,13)$.

Cell culture, transfection and treatment. The ovarian cancer cell lines were cultured in RPMI-1640 medium (Invitrogen, Burlington, ON, Canada) supplemented with $10 \%$ fetal bovine serum (FBS) and maintained at $37^{\circ} \mathrm{C}$, in $5 \% \mathrm{CO}_{2}$, as reported previously $(1,12,13)$. All tissue culture reagents were obtained from Sigma-Aldrich (St. Louis, MO, USA). The miR-136 mimics and inhibitors were designed and chemically synthesized by Ambion (38422-01; Life Technologies Corporation, Denmark).

Lipofectamine 2000 (Invitrogen) was incubated with pre-miR-136 (miRNA mimic), anti-miR-136 (inhibitor) or their scrambled negative controls (Ambion, mock) at a concentration of $90 \mathrm{nmol} / \mathrm{l}$ and incubated in serum-free RPMI-1640 for 20 min before being added to $\mathrm{C} 13$ or OV2008 cells, respectively. Cells were incubated at $37^{\circ} \mathrm{C}$ for $4 \mathrm{~h}$ before $10 \% \mathrm{FBS}$ was replaced. For cisplatin treatment, cells were maintained in medium with the desired doses of cisplatin (P4394; Sigma, USA).

miRNA microarray and data analysis. A microarray platform optimized for the analysis of a panel of 768 human miRNAs was used to analyze and compare the pattern of miRNA expression between CR and IR ( $\mathrm{n}=7$ for all) to platinum-based chemotherapy in EOC patients. Individual real-time quantitative polymerase chain reaction assays were formatted into a TaqMan low-density array (TLDA; Applied Biosystems), which was performed at the Shannon McCormack Advanced Molecular Diagnostics Laboratory Research Services, Dana Farber Cancer Institute, Harvard Clinic and Translational Science Center, Boston, MA, USA. The normalized microarray data were managed and analyzed by StatMiner ver. 3.0 (Integromics ${ }^{\mathrm{TM}}$ ).

Quantitative real-time PCR. Quantitative real-time PCR (qRT-PCR) was performed using the TaqMan MicroRNA Reverse Transcription kit (Applied Biosystems, Foster City CA, USA) on an Agilent Technologies Stratagent Mx3000P (USA). A $500 \mathrm{ng}$ mass of total RNA in $1 \mu \mathrm{l}$ of RNase-free water was used in $20 \mu \mathrm{l}$ of the RT mix. The following primer pairs were used: hsa-miR-136 (ABI miRNA-specific primers, Cat no. 4427012). The products were detected with SYBRGreen I, and their relative miRNA levels were calculated using the comparative $\mathrm{Ct}$ (cycle threshold, $2^{-\Delta \Delta \mathrm{Ct}}$ ) method with U6 as the endogenous control.

Cell growth and viability assays. Manual cell counting using trypan blue staining was used to determine the effect of cisplatin on the different cell lines. Briefly, cells were cultured in 96-well plates at a density of $1 \times 10^{3} /$ well for $48 \mathrm{~h}$ after transfection, and were then treated with $0,10,15,20,25,30,35,60,80$ and $120 \mu \mathrm{mol} / \mathrm{l}$ cisplatin (P4394; Sigma) for $48 \mathrm{~h}$. Subsequently, live-cell and dead-cell activities were detected by trypan blue staining. Cell surviving percentage was assessed as the ratio of live to total (live + dead) cells. Each point represents the mean \pm SD of triplicates.

Migration and invasion assays. For migration and invasion assays, $48 \mathrm{~h}$ after transfection, the cells were serum starved for $12 \mathrm{~h}$ before performing the assays. The assays were performed with BD Bio-Coat Control Insert Chambers, 24-well plates with an $8-\mu \mathrm{m}$ pore size, and BD Bio Coat Matrigel Invasion Chambers, respectively. For the wound-healing assay, $3 \times 10^{3}$ cells were plated in the top chamber in $0.5 \mathrm{ml} \mathrm{MEM} \mathrm{with}$ $0.5 \%$ FBS. The cells that did not migrate through the membrane were removed with a cotton swab. A wound was created using an aerosol P200 pipette tip after $12 \mathrm{~h}$ of incubation, and then microscopic images were captured at $0,8,16$ and $24 \mathrm{~h}$. In the Boyden chamber invasion assay, $3 \times 10^{3}$ cells were seeded into $8-\mu \mathrm{m}$ pore inserts coated with $50 \mu \mathrm{l}$ Matrigel (BectonDickinson). The bottom chamber, which contained $50 \%$ DMEM with $10 \%$ FBS and L-glutamine, was used as a chemoattractant. After $24 \mathrm{~h}$, the cells that had penetrated the Matrigel to invade the lower surface of the membrane were fixed in methanol and stained with crystal violet (1\%), as was described for the migration assay. Migrating or invading cells on the surface of the membranes were counted manually using Image-Pro Plus version 6.0 (Media Cybernetics, Inc.).

Cellular apoptosis assay with flow cytometry. Annexin V binding with propidium iodide (PI) staining was performed using the Annexin V-FITC Apoptosis Detection kit (KGA106; KeyGen Biotech, Nanjing, China). Ovarian cancer cells 
were initially seeded at a concentration of $1 \times 10^{5}$ cells $/ \mathrm{ml}$, in 6 -well plates, and incubated at $37^{\circ} \mathrm{C}$ in a humidified atmosphere with $5 \% \mathrm{CO}_{2}$ for $48 \mathrm{~h}$ after transfection. The cells were then treated with 50 and $25 \mu \mathrm{mol} / 1$ cisplatin, respectively. After exposure to the drugs for $48 \mathrm{~h}$, the cells were dissociated using $0.05 \%$ EDTA-free trypsin and washed with cold PBS. Approximately $1 \times 10^{6}$ cells were suspended in $100 \mu \mathrm{l}$ of Annexin $\mathrm{V}$ incubation reagent. After incubation in the dark for $20 \mathrm{~min}$ at room temperature, cellular fluorescence was analyzed by the BD FACSCalibur Flow Cytometer (BD Biosciences, USA) within $30 \mathrm{~min}$. Control tubes containing binding buffer only and cells containing Annexin V alone and PI alone were initially used to calibrate the instrument.

Comet assay. The single cell gel electrophoresis assay (also known as the Comet assay) is a sensitive technique for measuring DNA strand breaks at the level of the individual cell. This is a standard technique for evaluation of DNA biomonitoring, genotoxicity testing and damage/repair $(14,15)$. The assay was performed as described by Pérez et al (14) using a DNA damage detection kit (KeyGen Biotech), with some modifications. Exponentially growing cells were exposed to cisplatin $50 \mu \mathrm{mol} / \mathrm{l}$ (based on the $\mathrm{IC}_{50}$ assay) for $1 \mathrm{~h}$. The cells were washed and incubated at $37^{\circ} \mathrm{C}$ in $5 \% \mathrm{CO}_{2}$ for $48 \mathrm{~h}$. Next, the cells were trypsinized and resuspended in $1 \mathrm{ml}$ culture medium. After that, $10 \mu \mathrm{l}$ of the cells $\left(1 \times 10^{4}\right)$ was encapsulated in $75 \mu \mathrm{l}$ of $0.7 \%$ low-melting-point agarose at $37^{\circ} \mathrm{C}$. This mixture was layered onto precoated slides with $0.5 \%$ standard agarose and covered with a coverslip. The agarose was allowed to solidify for $10 \mathrm{~min}$ at $4^{\circ} \mathrm{C}$, and then the coverslip was gently removed. The slides were then immersed in a precooled lysis buffer (cat. no. KGA240) for at least $90 \mathrm{~min}$ at $4^{\circ} \mathrm{C}$ and then incubated in an alkaline buffer $(1 \mathrm{mmol} / 1$ EDTA and $300 \mathrm{mmol} / 1 \mathrm{NaOH} ; \mathrm{pH}>13.0$ ) for $60 \mathrm{~min}$ at room temperature to allow for DNA unwinding and alkali-labile site expression. Electrophoresis was conducted in the same alkaline buffer for $30 \mathrm{~min}$ at $25 \mathrm{~V}(0.86 \mathrm{~V} / \mathrm{cm})$ and $300 \mathrm{~mA}$. After electrophoresis, the slides were washed at least three times with $0.4 \mathrm{mmol} / 1 \mathrm{Tris}-\mathrm{HCl}(\mathrm{pH} 7.5)$ prior to staining with propidium iodide (PI) $(20 \mu \mathrm{l})$ (KeyGen) for $10 \mathrm{~min}$. Comet tails were visualized using a FITC filtered fluorescence microscope. In these experiments, cells with high amounts of cisplatininduced cross-links have shorter tails compared to cells in which cross-links have been repaired effectively. This can be explained by the fact that unrepaired platinum-induced DNA cross-links will stay together after DNA degradation, resulting in larger DNA fragments (16). The tail length is proportional to the DNA repair. This is followed by visual analysis with staining of DNA and calculating the percentage of DNA in comet tails, tail length (in $\mu \mathrm{m}$ ), tail moment (TM) and olive tail moment (OTM) to determine the extent of DNA damage/ repair (CASP ver. 1.2.3beta2, Krzysztof Konca; casplab.com).

Statistical analysis. SPSS 17.0 software (Chicago, IL, USA) was applied for all quantitative analyses in the study, except for the microarray data. Data are expressed as arithmetic means \pm $\mathrm{SD}$ of the number (n) of experiments. Samples were analyzed with repeated measures analysis of variance, and differences in the incidences were analyzed using ANOVA. The images of comet assay were analyzed using Image-Pro Plus version 6.0.
Table I. Clinicopathological characteristics of the ovarian cancer patients.

\begin{tabular}{lcr}
\hline & \multicolumn{2}{c}{ Clinical response } \\
\cline { 2 - 3 } Characteristics & $\begin{array}{c}\text { Complete } \\
(\mathrm{n}=19)\end{array}$ & $\begin{array}{c}\text { Incomplete } \\
(\mathrm{n}=15)\end{array}$ \\
\hline Mean age (years) & 52.7 & 55.6 \\
Mean serum CA-125 (m/ml) & & \\
Before platinum treatment & 796.5 & $1,533.8^{\mathrm{b}}$ \\
After platinum treatment & 22.7 & $416.5^{\mathrm{b}}$ \\
Mean survival time (months) & 57.2 & $35.8^{\mathrm{a}}$ \\
Mortality rate (\%) & 31.6 & $62.3^{\mathrm{b}}$ \\
\hline
\end{tabular}

Compared with the complete response (CR) group, ${ }^{\mathrm{a}} \mathrm{P}<0.05$, ${ }^{\mathrm{b}} \mathrm{P}<0.01$.

$\mathrm{P}<0.05$ was considered to indicate a statistically significant difference.

\section{Results}

Patient characteristics. Nineteen EOC patient samples were classified as CR and 15 as IR to primary platinum-based therapy following surgery, and FFPE blocks were obtained after surgery. The clinicopathological characteristics of the patients who provided the OPSC samples are listed in Table I. The mean serum CA-125 level in the IR patients was significantly higher than that in the patients with $\mathrm{CR}(\mathrm{P}<0.001$ for all).

microRNA microarray results and $q R T-P C R$ validation. To further characterize the unique miRNAs in OPSC differentiation, specimens from the CR and IR OPSC patients were initially analyzed by miRNA microarray, respectively. Of the 768 miRNAs analyzed by microarray, we identified that miR-136 expression was strongly downregulated in the IR OPSC when compared with the CR OPSC patients (Fig. 1A). In order to confirm the microarray results, qRT-PCR validation was performed. RNA was isolated from a new set of FFPE tissues to increase the likelihood that the observed differences in miRNA expression profiles represent biologically significant changes. Consistent to the microarray results, miR-136 was weakly expressed in the IR OPSC with significance, and representative analysis is shown in Fig. 1B.

miR-136 expression in the different chemoresistant ovarian cancer cell lines; overexpression of miR-136 decreases cell survival of a chemoresistant cisplatin cell line. We demonstrated that the miR-136 expression level was significantly higher in the CR tumor specimens than the level in the IR tumor specimens (Fig. 1A and B). Thus, we chose to further verify the relationship between miR-136 expression and chemosensitivity using the human ovarian cancer cell lines OV2008 and A2780S and the cisplatin-resistant variants (C13 and A2780CP, respectively). In Fig. 1C, significant downregulation of miR-136 expression was noted in the $\mathrm{C} 13$ and A2780CP cell lines compared with their cisplatin-sensitive 

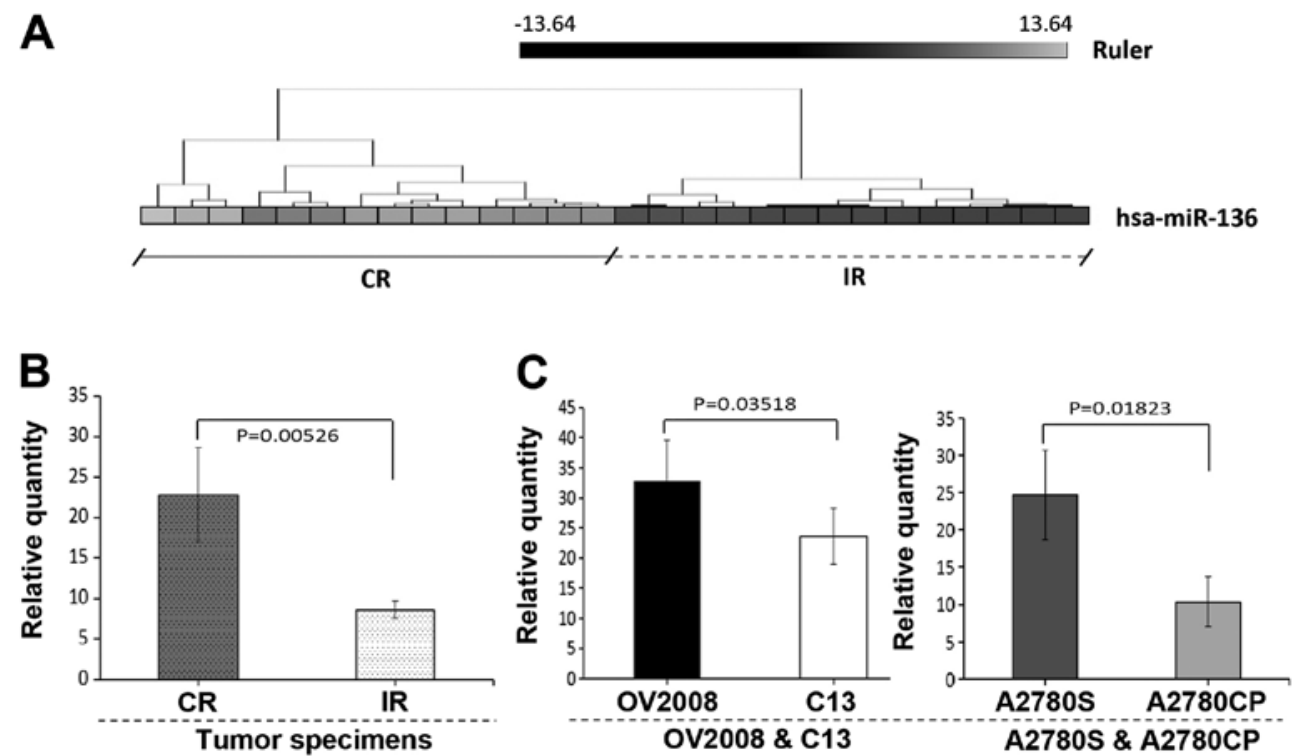

D

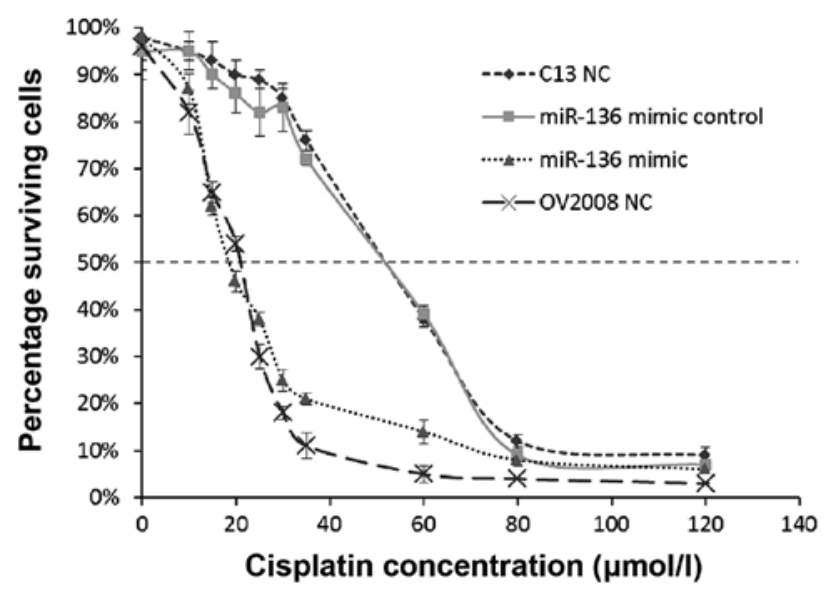

Figure 1. miR-136 expression is significantly decreased in EOC patient tissue samples and cell lines with chemotherapy resistance. (A) Hierarchical clustering of miRNA microarray analysis between ovarian cancer samples with complete response (CR) and incomplete response (IR). miR-136 is weakly expressed with statistical significance $(\mathrm{P}<0.0001)$ in the IR group vs. the CR group. (B) Validation of miR-136 expression in the tumor specimens by qRT-PCR. The result is consistent with the microarray results. (C) In ovarian cancer cell lines, we found that miR-136 expression was lower in the cisplatin-resistant parental cell lines (C13 and A2780CP) than their cisplatin-sensitive variants (OV2008 and A2780S). $\mathrm{P}=0.03518$ and $\mathrm{P}=0.001823$, respectively. (D) Trypan blue staining assay to determine cisplatin sensitivity in C13, its cisplatin-sensitive variant OV2008 and the two transfected cell lines (C13-miR-136 mimic and C13-miR-136 mimic control). Growth inhibition was determined using the trypan blue staining assay and the percentage of surviving cells was calculated as described. All results are the mean of at least three independent experiments and error bars show the standard deviation of the mean.

variants. These results are consistent with those for the clinical OVC patient specimens.

Furthermore, to test whether miR-136 is causally related to cellular sensitivity to cisplatin, we conducted transfection experiments with cisplatin-resistant ovarian cancer cells. Fig. 1D shows the sensitivity to cisplatin of the C13 cells, its cisplatin-sensitive variant OV2008 and the two transfected cell lines (C13+miR-136 mimic and C13+miR-136 mimic control). The drug concentration that inhibited cell growth by $50 \%\left(\mathrm{IC}_{50}\right.$ values) in the cisplatin-resistant ovarian cancer cells (C13) was 2.5-fold higher than that for its parental cell line (OV2008). $\mathrm{C} 13$ and $\mathrm{C} 13+\mathrm{miR}-136$ mimic control showed similar $\mathrm{IC}_{50}$ values, indicating that the transfection itself had no effect on cell growth. In contrast, the miR-136-overexpressing cells, C13+ miR-136 mimic and OV2008, showed substantial sensitivity to cisplatin. The $\mathrm{IC}_{50}$ values for these cells were $\sim 2.5$-fold lower compared to the $\mathrm{C} 13$ cell line $(\mathrm{P}<0.01$; ANOVA test).
No difference was found in $\mathrm{IC}_{50}$ values between $\mathrm{C} 13+\mathrm{miR}-136$ mimic and OV2008 cells. These results indicate that miR-136 influences the cellular sensitivity to cisplatin.

miR-136 has no effect on cell migration and invasion but modulates cisplatin-induced apoptosis. The progression of a malignant tumor is determined by the metastatic and invasive properties of the tumor cells, which promote malignant cells to invade the extracellular matrix and metastasize to distant sites. To further clarify the role of miR-136 in the progression of ovarian cancer with primary chemotherapy, a commercially available miR-136 mimic was applied to the cisplatin-resistant parental cell line $(\mathrm{C} 13)$ for a series of migration and invasion experiments. Fig. 2A and B shows that the speed of migration in the $\mathrm{C} 13$ cells had no significant change when compared to their own mock-transfected and NC cells after overexpression of miR-136. The slope of the general linear model and number 
A

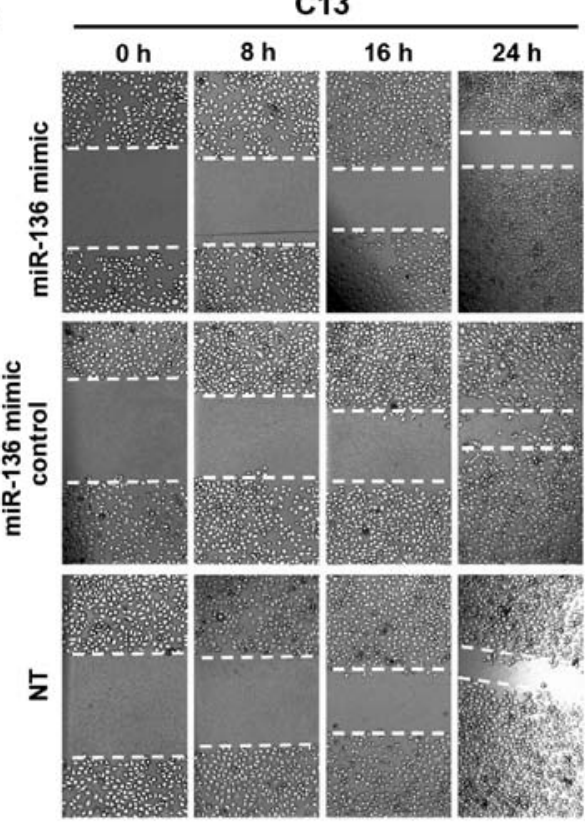

B

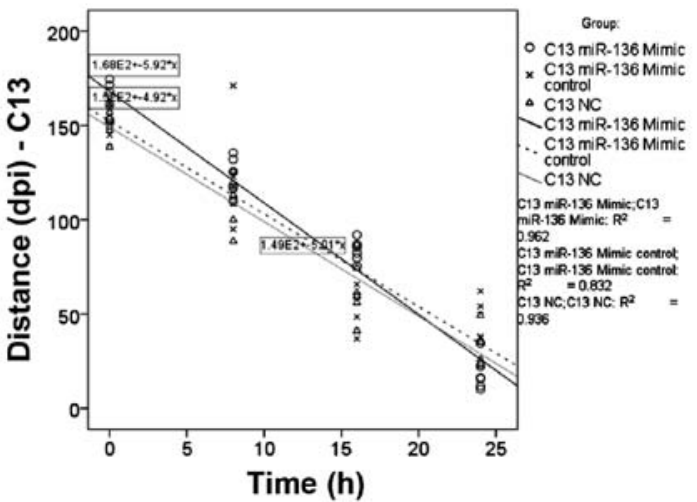

C
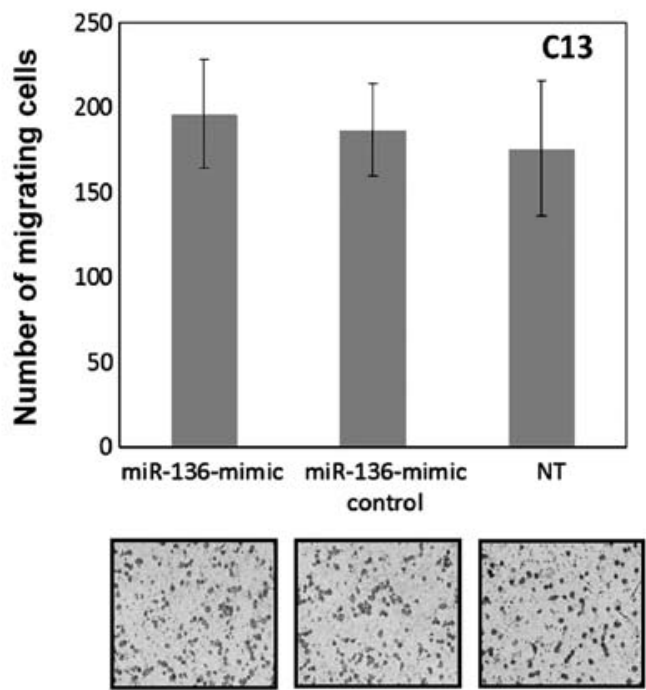

D

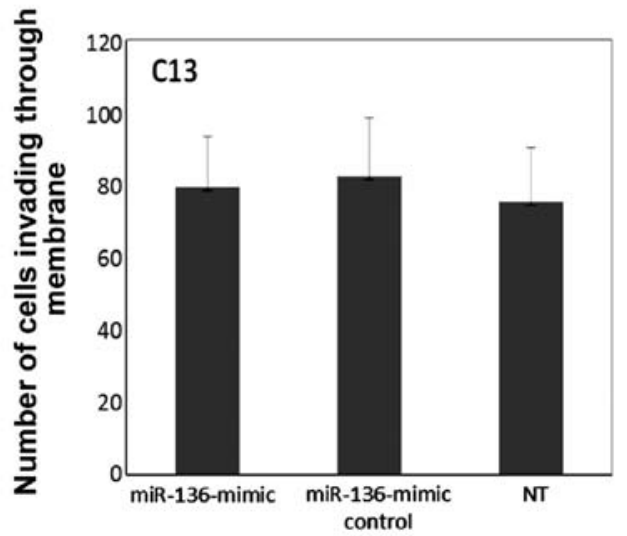

Figure 2. Overexpression of miR-136 has no effect on cell migration and invasion in the chemoresistant OVC cell line C13. (A) C13 cells were transfected with miR-136-mimic, miR-136-mimic control (mock) and negative control, respectively. After 48 h, wounds were inflicted and images were captured at 0 , 8 , 16 and $24 \mathrm{~h}$ after wounding. Lines indicate the width of the wound at each time-point. Images shown are from one experiment representative of six separate experiments. (B) The wound-healing tendency (slope) of the miR-136-mimic-treated C13 cells had no significant change when compared with their own mocktransfected and NC cells, respectively. (C) miR-136 transfection was carried out to assess cell motility using quantitative Boyden chamber assay. After 48 h, the miR-136-mimic-transfected C13 cells showed no significant decline in motility when compared to the mock-transfected and the negative-control cells, per vision-field at x100. (D) The number of cells able to invade through the Matrigel-coated Boyden chambers was also determined for each group. Columns, mean number of cells from six replicates; bars, $\mathrm{SD}$. Compared with either negative control or mock-transfected cells ( $\mathrm{P}>0.05 \mathrm{for}$ all).

of migrating cells within a certain period was used to reflect the motility potential. Using quantitative Boyden chamber analysis, the motility of the $\mathrm{C} 13$ cells was not reduced after overexpression of miR-136 (Fig. 2C and D).

Cisplatin and other platinum-based cancer drugs bind to double-stranded DNA and form DNA adducts, interfering with DNA replication and RNA transcription ultimately triggering apoptosis. Thus, we examined whether miR-136 is involved in cisplatin-induced chemoresistance by affecting apoptosis. As expected, the proportion of apoptotic cells was significantly increased in the $\mathrm{C} 13+\mathrm{miR}-136-$ mimic compared to its own NC- or mock-transfected group. The cisplatin-induced apoptosis in the C13+miR-136-mimic cells was comparable with the apoptosis of the OV2008 cells. These results indicated that the proapoptotic activity in the cisplatin-resistant $\mathrm{C} 13$ cell line was restored by introducing miR-136 (Fig. 3).
miR-136 is essential for repair of cisplatin-induced DNA damage in vitro. To validate whether the high DNA repair ability of ovarian cancer cells following cisplatin treatment is caused by a defect in miR-136, we evaluated cisplatin-induced DNA damage using the COMET assay, in C13 cells, its cisplatin-sensitive variant (OV2008), and the transfected variants C13+miR-136 mimic and OV2008+miR-136 inhibitor. In order to quantify the DNA damage/repair process, we measured the percentage of DNA in comet tails, tail length (in $\mu \mathrm{m}$ ), TM and OTM for each gel. Fig. 4A and Table II depict the decreasing trend of DNA damage measured with CASP ver. 1.2.3beta2. The DNA damage, as measured manually, is shown in Fig. 4B. As expected, tails of the DNA damage/ repair deficient OV2008 cell line were smaller than the $\mathrm{C} 13$ tails. After transfection with miR-136, the comet tails in the C13+miR-136 mimic were also shorter than the C13 tails. 

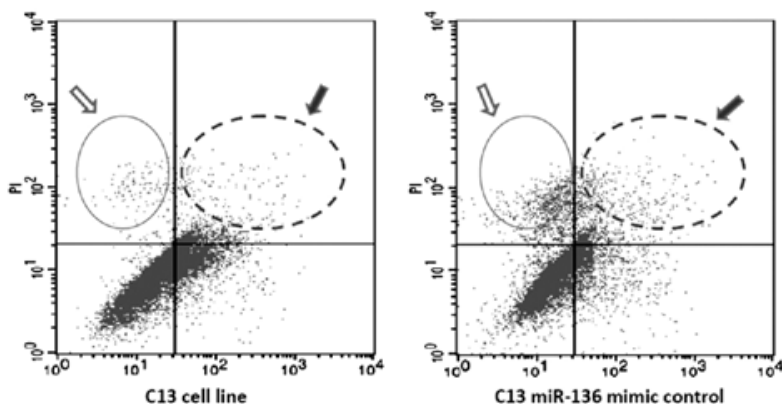

C13 miR-136 mimic control
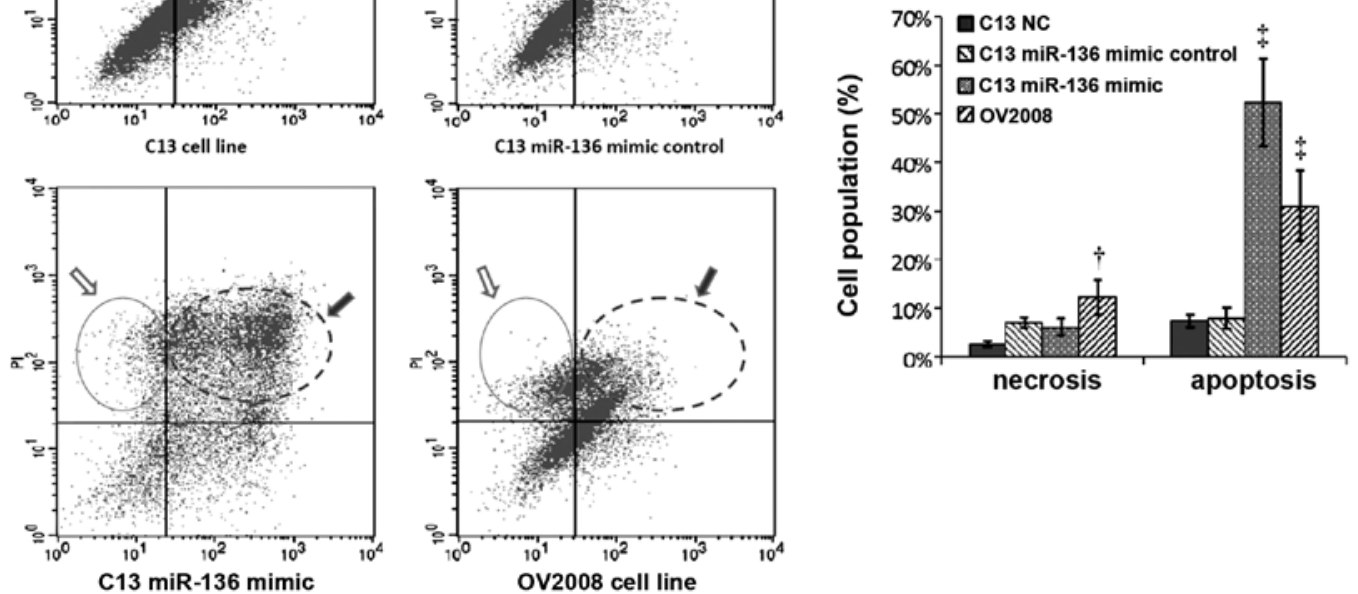

Figure 3. High level of miR-136 suppresses ovarian cancer cell resistance to cisplatin-induced apoptosis by flow cytometric assays. Flow cytometric detection of apoptosis via Annexin V-FITC/PI staining in C13 cells, which are cisplatin-resistant, following treatment with cisplatin for $48 \mathrm{~h}$ after transfection with miR-136 mimic or negative control. The percentage of apoptosis in the miR-136-transfected C13 cells was significantly increased whwn compared with its own NC- or mock-transfected group, and was even comparable with the OV2008 cells. Meanwhile, the percentage of OV2008 necrotic cells was higher than that in the other groups. Solid area represents necrosis (hollow arrow), and dotted area represents apoptosis (solid arrow). Compared with the NC- or mock-transfected $\mathrm{C} 13$ cells, ${ }^{\dagger} \mathrm{P}<0.05,{ }^{+} \mathrm{P}<0.001$
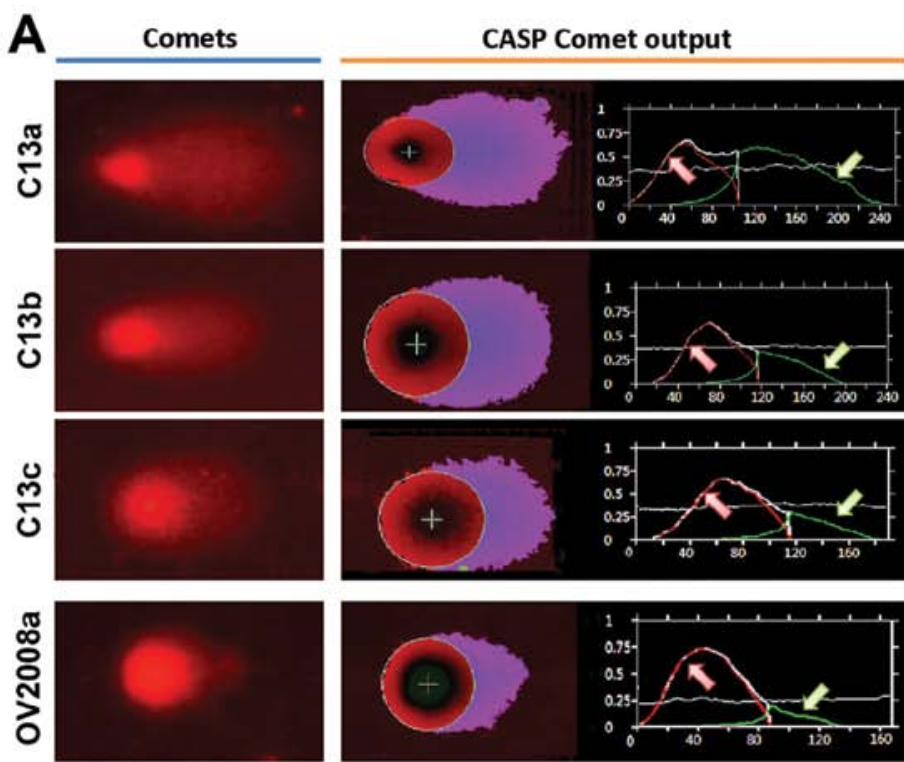

$\mathbf{B}$
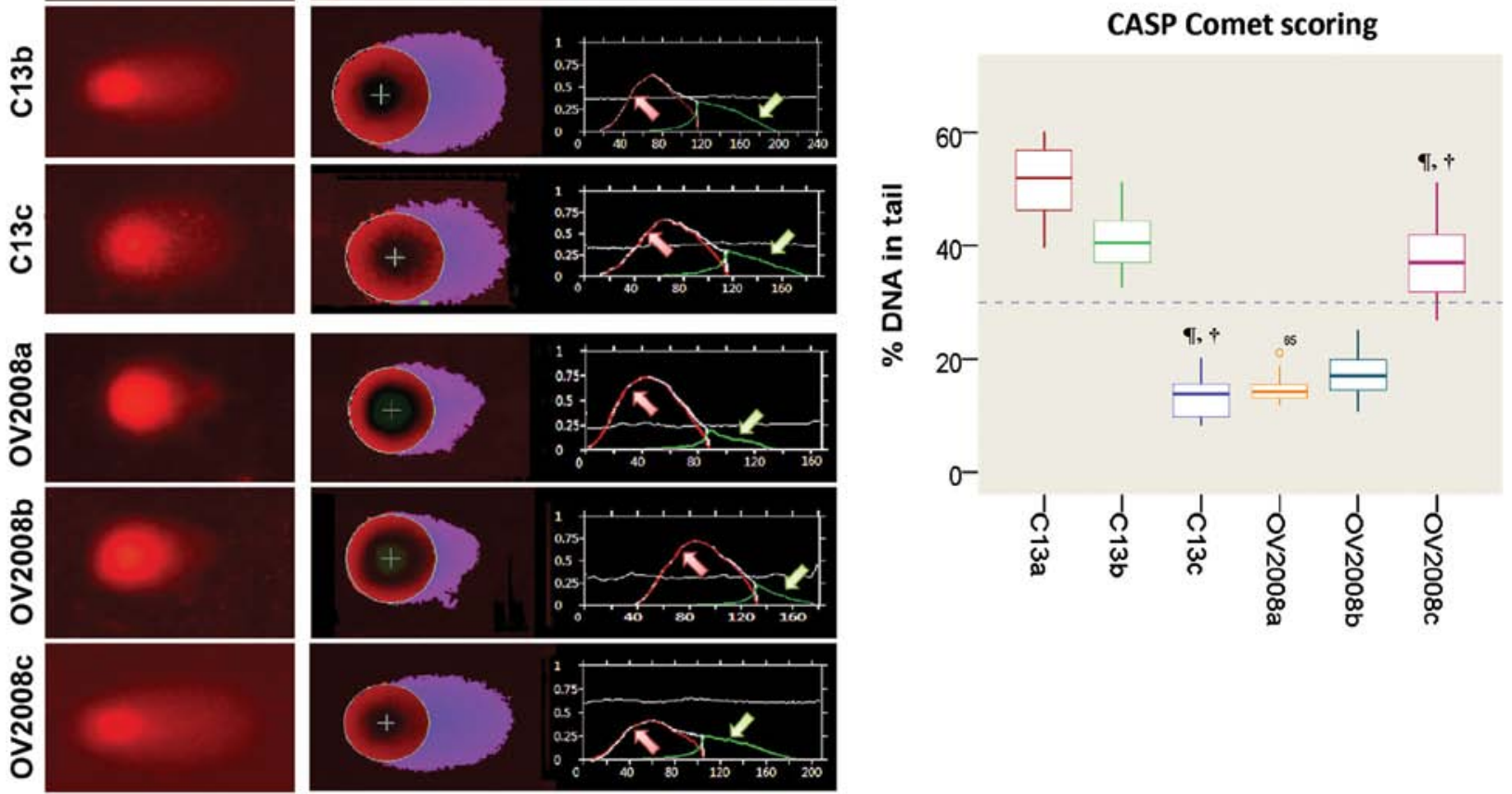

Figure 4. CASP comet analysis of alkaline comet assay images. We used CASP comet to analyze alkaline comet assay images of individual standard damaged cells of each group after treatment with cisplatin. (A) CASP Comet analysis. The left column is the alkaline comet assay image of each group, and the right column is the output and analysis of the CASP comet. Red line (area indicated by red arrow) represents the head of the comet, and the green line (area indicated by green arrow) represents the area of the tail of the comet. (B) Trend of the amount of DNA damage across C13 and OV2008 cell lines. C13a, C13 NC; C13b, C13+miR-136 mimic control; C13c, C13+miR-136 mimic; OV2008a, OV2008 NC; OV2008b, OV2008+miR-136 inhibitor control; OV2008c, OV2008+miR-136 inhibitor. Compared with the normal control (NC) group of their own cell line, ${ }^{9} \mathrm{P}<0.001$; compared with their scrambled negative controls, ${ }^{, \mathrm{P}}<0.001$. 
Table II. Spontaneous and cisplatin-induced mean values and their standard deviation of four replicates of the Comet assay parameters after treatment with cisplatin.

\begin{tabular}{llcccc}
\hline $\begin{array}{l}\text { Ovarian cancer } \\
\text { cell lines }\end{array}$ & \multicolumn{1}{c}{ Transfection status } & \% Tail DNA \pm SD & Tail length \pm SD & TM \pm SD & OTM \pm SD \\
\hline C13 & Normal control & $51.62 \pm 5.80$ & $133.95 \pm 22.43$ & $70.66 \pm 11.61$ & $50.10 \pm 5.36$ \\
& miR-136 mimic control & $41.34 \pm 5.13$ & $100.25 \pm 26.55$ & $51.30 \pm 11.81$ & $44.57 \pm 12.28$ \\
& miR-136 mimic & $13.33 \pm 3.19^{\mathrm{a}, \mathrm{b}}$ & $46.50 \pm 7.73^{\mathrm{a}, \mathrm{c}}$ & $9.87 \pm 2.82^{\mathrm{a}, \mathrm{c}}$ & $10.07 \pm 3.60^{\mathrm{a}, \mathrm{c}}$ \\
OV2008 & Normal control & $14.61 \pm 2.19$ & $45.90 \pm 5.45$ & $8.52 \pm 1.82$ & $8.18 \pm 1.50$ \\
& miR-136 inhibitor control & $17.17 \pm 3.90$ & $52.95 \pm 9.76$ & $10.15 \pm 2.63$ & $9.77 \pm 2.60$ \\
& miR-136 inhibitor & $36.98 \pm 6.16^{\mathrm{a}, \mathrm{b}}$ & $98.45 \pm 19.59^{\mathrm{a}, \mathrm{b}}$ & $24.67 \pm 4.88^{\mathrm{a}, \mathrm{c}}$ & $24.82 \pm 6.76^{\mathrm{a}, \mathrm{c}}$
\end{tabular}

Compared with the normal control (NC) group of their own cell line, ${ }^{a} \mathrm{P}<0.001$; compared with their scrambled negative controls, ${ }^{b} \mathrm{P}<0.001$ and ${ }^{\mathrm{c}} \mathrm{P}<0.0001$. SD, standard deviation; TM, tail moment; OTM, olive tail moment.
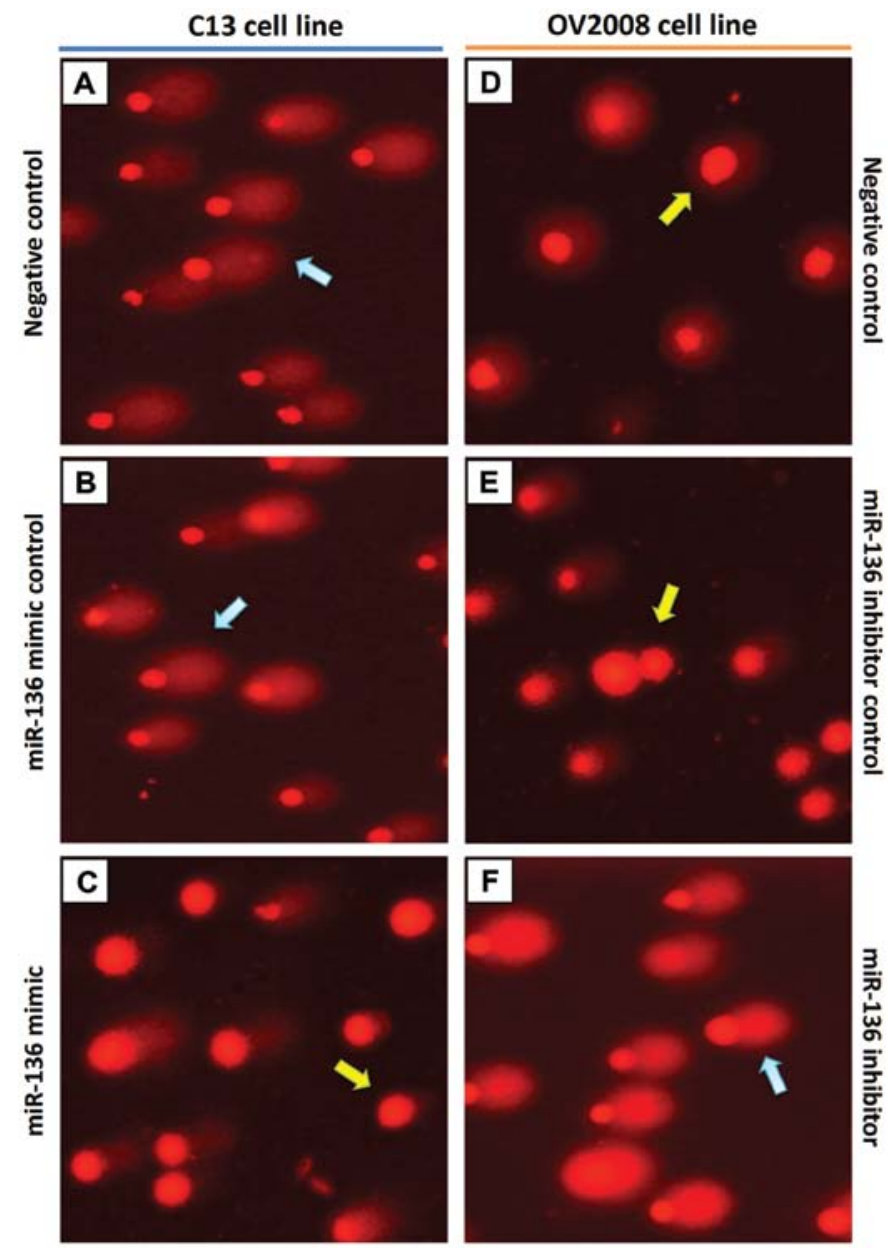

Figure 5. Representative image of the modified COMET assay used for interpretation of remaining DNA damage after treatment with cisplatin. (A) C13 negative control. (B) C13 cells transfected with miR-136 mimic control. (C) C13 cells transfected with miR-136 mimic. (D) OV2008 negative control. (E) OV2008 cells transfected with miR-136 inhibitor control. (F) OV2008 cells transfected with miR-136 inhibitor. Light blue arrows indicate comets with a long tail, and yellow arrows indicate the comets with a short tail.

On the contrary, the miR-136 inhibitor increased the tail size compared with that of the OV2008 cells. These results indicate that DNA repair activity in the cisplatin-resistant ovarian cancer cells was fully restored by downregulation of miR-136 (Fig. 4). A representative image of the modified COMET assay used for data analysis is shown in Fig. 5.

\section{Discussion}

Due to the heterogeneity and complexity of cancer cells and the different mechanism of action of anticancer drugs, several major mechanisms have been demonstrated to play a crucial role in the resistance of chemotherapy, such as insensitivity 
to drug-induced apoptosis, increased DNA repair, decreased drug accumulation, and induction of drug-detoxifying mechanisms $(17,18)$. Most previous studies focused on genetic and epigenetic alterations occurring during tumor formation and progression. However, recent data have demonstrated that drug resistance is regulated not only by genetic and epigenetic changes, but also by miRNAs (17). In this context the recent identification of the regulatory role of miRNAs extended the spectrum of possible key factors involved in such a processes.

The advent of microarray technologies offers the opportunity to identify novel resistance mechanisms in clinically relevant material, e.g. tumor tissue from patients before and after chemotherapy. The discovery of a set of miRNAs strongly deregulated in drug-resistant patients will open an avenue for innovative cancer treatment. In a recent work, Shen et al (19) reported that miR-136 expression was found to be deregulated in a human non-small cell lung cancer cell line. In addition, it has been found that miR-136 is downregulated in human glioma, and that miRNA promotes apoptosis of glioma cells by targeting AEG-1 and Bcl-2 (19). However, the roles of miR-136 in OVC are still largely unknown.

Here, we found that miR-136 was markedly downregulated in cisplatin-resistant EOC patient tissues and in cell lines. Upregulation of miR-136 inhibited EOC cell survival, and promoted cell apoptosis. In fact, miRNAs can alter the cellular response to a specific drug or class of drugs via many mechanisms other than survival or apoptotic signaling, such as by interfering with drug targets and DNA repair (17). In this study, besides regulating cytotoxic drug-induced apoptosis, miR-136 might also alter the cellular response to cisplatininduced DNA excision. The levels of adducts corrected with their extent of DNA damage/repair, in terms of the percentage of DNA in comet tails, tail length, TM and OTM, revealed that miR-136 is essential for repair of cisplatin-induced DNA damage. Yet, we found no association between miR-136 and migration or invasion potential in the ovarian cancer cell lines.

The antitumour effect of cisplatin results from platinum DNA cross-links, and ultimately leads to programmed cell death. However, several cellular DNA repair mechanisms are capable of repairing damage from platinum-DNA adducts, such as the nucleotide excision repair (NER) system (20). In summary, in this study we demonstrated that a specific miRNA, miR-136, is deregulated in cisplatin-resistant ovarian cancer, and is also involved in platinum-resistance by affecting DNA repair and cell apoptosis. We believe that the approach described here, using microarray technologies that predict primary chemotherapy response, coupled with expression data that identify oncogenic pathway deregulation to reveal mechanisms of cisplatin-resistance, represents an important step toward the goal of personalized cancer treatment, including EOC therapy.

\section{Acknowledgements}

This research was supported by the National Basic Research Program of China 973 Program No. 2012CB517600 (No. 2012CB517603), and a MOST grant (2008 DFA30720) to C.S.

\section{References}

1. Zhao H, Ding Y, Tie B, et al: miRNA expression pattern associated with prognosis in elderly patients with advanced OPSC and OCC. Int J Oncol 43: 839-849, 2013.

2. Matei DE and Nephew KP: Epigenetic therapies for chemoresensitization of epithelial ovarian cancer. Gynecol Oncol 116: 195-201, 2010.

3. Yu X, Zhang X, Bi T, et al: MiRNA expression signature for potentially predicting the prognosis of ovarian serous carcinoma. Tumour Biol 34: 3501-3508, 2013.

4. Sorrentino A, Liu CG, Addario A, Peschle C, Scambia G and Ferlini C: Role of microRNAs in drug-resistant ovarian cancer cells. Gynecol Oncol 111: 478-486, 2008.

5. Chen Y,Ke G,Han D, Liang S, Yang G and Wu X: MicroRNA-181a enhances the chemoresistance of human cervical squamous cell carcinoma to cisplatin by targeting PRKCD. Exp Cell Res 320: 12-20, 2014.

6. Iorio MV and Croce CM: microRNA involvement in human cancer. Carcinogenesis 33: 1126-1133, 2012.

7. Meng F, Henson R, Lang M, et al: Involvement of human micro-RNA in growth and response to chemotherapy in human cholangiocarcinoma cell lines. Gastroenterology 130: 2113-2129, 2006.

8. Yang H, Kong W, He L, et al: MicroRNA expression profiling in human ovarian cancer: miR-214 induces cell survival and cisplatin resistance by targeting PTEN. Cancer Res 68: 425-433, 2008.

9. Passetti F, Ferreira CG and Costa FF: The impact of microRNAs and alternative splicing in pharmacogenomics. Pharmacogenomics J 9: 1-13, 2009.

10. Ratner ES, Keane FK, Lindner R, et al: A KRAS variant is a biomarker of poor outcome, platinum chemotherapy resistance and a potential target for therapy in ovarian cancer. Oncogene 31: 4559-4566, 2012.

11. Rubatt JM, Darcy KM, Tian C, et al: Pre-treatment tumor expression of ERCC1 in women with advanced stage epithelial ovarian cancer is not predictive of clinical outcomes: a Gynecologic Oncology Group study. Gynecol Oncol 125: 421-426, 2012.

12. Rustin GJ, Nelstrop AE, Bentzen SM, Piccart MJ and Bertelsen K: Use of tumour markers in monitoring the course of ovarian cancer. Ann Oncol 10: 21-27, 1999.

13. Rustin GJ, Nelstrop AE, McClean P, et al: Defining response of ovarian carcinoma to initial chemotherapy according to serum CA 125. J Clin Oncol 14: 1545-1551, 1996.

14. Pérez C, Díaz-García CV, Agudo-López A, et al: Evaluation of novel trans-sulfonamide platinum complexes against tumor cell lines. Eur J Med Chem 76: 360-368, 2014.

15. Gyori BM, Venkatachalam G, Thiagarajan PS, Hsu D and Clement MV: OpenComet: An automated tool for comet assay image analysis. Redox Biol 2: 457-465, 2014.

16. van Huis-Tanja LH, Kweekel DM, Lu X, Franken K, et al: Excision Repair Cross-Complementation group 1 (ERCC1) C118T SNP does not affect cellular response to oxaliplatin. Mutat Res Fundam Mol Mech Mutagen 759: 37-44, 2014.

17. Giovannetti E, Erozenci A, Smit J, Danesi R and Peters GJ: Molecular mechanisms underlying the role of microRNAs (miRNAs) in anticancer drug resistance and implications for clinical practice. Crit Rev Oncol Hematol 81: 103-122, 2012.

18. Li Z, Hu S, Wang J, Cai J, Xiao L, Yu L and Wang Z: MiR-27a modulates MDR1/P-glycoprotein expression by targeting HIPK2 in human ovarian cancer cells. Gynecol Oncol 119: 125-130, 2010.

19. Shen S, Yue H, Li Y, Qin J, Li K, Liu Y and Wang J: Upregulation of miR-136 in human non-small cell lung cancer cells promotes Erk1/2 activation by targeting PPP2R2A. Tumour Biol 35: 631-640, 2014

20. Kweekel DM, Gelderblom H and Guchelaar HJ: Pharmacology of oxaliplatin and the use of pharmacogenomics to individualize therapy. Cancer Treat Rev 31: 90-105, 2005. 